\title{
ADI 1050-6 Mechanical Behavior at Different Strain Rates and Temperatures
}

\author{
Andrew Ruggiero ${ }^{1, \mathrm{a}^{*}}$, Gianluca lannitti ${ }^{1, \mathrm{~b}}$, Stefano Masaggia ${ }^{2, \mathrm{c}}$ \\ and Federico Vettore ${ }^{2, \mathrm{~d}}$ \\ ${ }^{1}$ DICeM, University of Cassino and SL, Via G. Di Biasio 43 - 03043 Cassino, Italy \\ 2Zanardi Fonderie SpA, via Nazionale 3 - 37046 Minerbe (VR), Italy \\ aa.ruggiero@unicas.it, bg.iannitti@unicas.it, cMST@zanardifonderie.com, \\ dvfe@zanardifonderie.com
}

Keywords: Austempered ductile irons, high strain rate, constitutive modeling, continuum damage mechanics.

\begin{abstract}
An experimental characterization of the austempered ductile iron ISO 17804/JS/1050-6/S was performed carrying out tensile tests under different strain rates, temperatures and stress triaxiality levels. Then, composing a yield function surface, a hardening relation, and a damage criterion, a constitutive model was developed to describe the salient features of the observed macroscopic response. In particular, the Mohr-Coulomb yield function was selected to account for the pressure effect observed on the yield surface. A new hardening relation was proposed in order to account for both strain rate and temperature effects. The Bonora's damage model, developed in the framework of the continuum damage mechanics, was adopted to capture the failure condition under different stress triaxiality levels. The damage model was appropriately modified to account for the effect of strain rate and temperature on the failure strain.
\end{abstract}

\section{Nomenclature}

$\dot{\varepsilon} \quad$ Equivalent plastic strain rate

$\dot{D} \quad$ Damage rate

$\lambda \quad$ Coefficient of internal friction

$\nu \quad$ Poisson ratio

$\sigma \quad$ Equivalent stress

$\varepsilon \quad$ Equivalent accumulated plastic strain

$D \quad$ Damage varable

$E \quad$ Young's modulus

$f \quad$ Deviatoric yield function

$I_{1} \quad$ First principal invariant of stress

$J_{2} \quad$ Second stress deviator invariant

$R_{\nu} \quad$ Triaxiality function

$T \quad$ Absolute temperature

$T_{m} \quad$ Absolute melting temperature

\section{Introduction}

Austempered ductile irons (ADIs) combine the properties of high mechanical strength, wear and fatigue resistance with good ductility and low density. These properties allow ADIs to be a suitable alternative to high-strength steels. Nevertheless, the concern about a low ductility under dynamic loads often leads designers to exclude ductile cast irons for structural applications. In some applications, the high Charpy impact resistance requested for a structural material precludes using ADIs. Although, the 
energy absorbed in the test can be only used in a comparative approach and does not provide an allowable value for failure analysis, arbitrary values of this parameter are imposed as mandatory limits, just mimicking standard practices valid for steels [1].

In contrast with this perception, preliminary results from dynamic tensile tests show larger failure strain with respect to quasi-static data. This work was aimed to extend the knowledge on the ADIs performances providing reliable data on its mechanical behavior under different levels of strain rates, temperatures and stress triaxiality.

Data analysis was performed with the final purpose to arrive at the formulation of a simple and complete constitutive model for describing the strength and predicting failure of the material under the investigated loading conditions. The experimental results showed that, in addition to the interaction between graphite nodules and matrix, the mechanical behavior of the ADI 1050-6 is influenced by the single allotropic phases of the matrix. The originated complex behavior was addressed, from a phenomenological point of view, by combining the Mohr-Coulomb yield function, a new hardening relation, and a modified version of the Bonora's damage model. Once the constitutive model was defined, the material dependent coefficients have been determined by a reverse calibration procedure.

\section{Material and Experiments}

The material investigated in this work is the ADI ISO 17804/JS/1050-6/S, supplied by Zanardi Fonderie SpA. The material was cast in cylinders of $25 \mathrm{~mm}$ diameter and $200 \mathrm{~mm}$ long. The material microstructure showed a nodularity higher than $90 \%$ and equally spaced spheroids with an average spheroid diameter of $40 \mu \mathrm{m}$, Fig. 1. The austempering heat treatment produces a matrix microstructure consisting of acicular ferrite, which has a body-centered cubic (BCC) crystal structure, in carbon-enriched austenite, with a face-centered cubic (FCC) structure.

Specimens were machined as smooth bars, with a length of reduced section of $14 \mathrm{~mm}$, and round notched specimens, with notch radii of 1.5, 3.0, $6.0 \mathrm{~mm}$, labeled as RndA, RndB, and RndC respectively. The same minimum section, with a diameter of $3.5 \mathrm{~mm}$, was used for all the specimens in order to avoid any possible scale effect.

Quasi-static tensile tests were performed at the strain rate of $0.001 \mathrm{~s}^{-1}$, at three different temperatures $(213,298$, and $343 \mathrm{~K})$ with an electromechanical testing machine Instron 5586. At room temperature, strain was measured using an extensometer with a reference length of $12.5 \mathrm{~mm}$ and with digital image correlation (DIC) technique. At the other temperatures, only load and stroke displacement were recorded.

Dynamic uniaxial tests were carried out using a direct tension split Hopkinson pressure bar (DTSHPB) at two temperature (RT and $213 \mathrm{~K}$ ) and two nominal strain rates (740 and $1200 \mathrm{~s}^{-1}$ ). The engineering stress-strain response was obtained by the bar signals. For the test at room temperature,

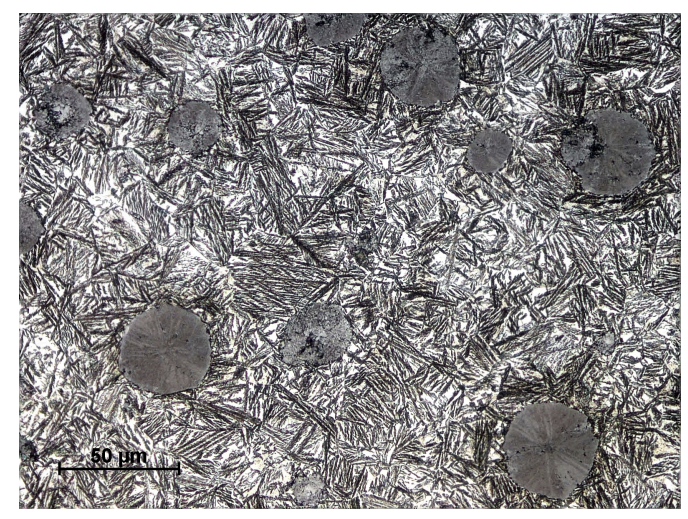

Fig. 1 ADI JS/1050-6 microstructure 
a high speed camera was also used for the DIC technique. More details about experimental setup can be found in [3].

The comparisons of the uniaxial tensile tests at different temperatures (Fig. 2), in both quasistatic and dynamic regimes, show that the work hardening rate decreases with temperature, as usual for FCC structures, whereas the apparent yield strength seems to increase slightly. On the contrary, comparisons of Fig. 3 show that strain rate affects the material yield stress and not the hardening rate, similar to what occurs for BCC structures [2].

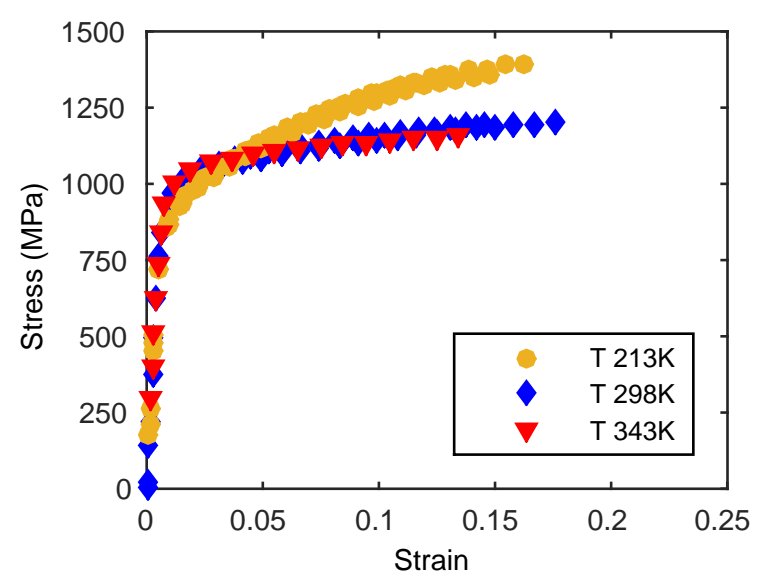

(a)

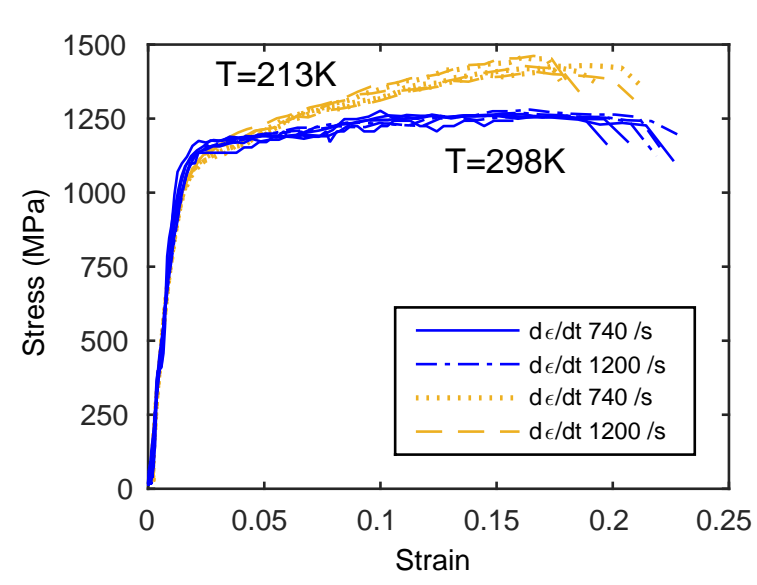

(b)

Fig. 2 Stress-strain curves at different temperatures: (a) quasistatic regime; (b) dynamic regime

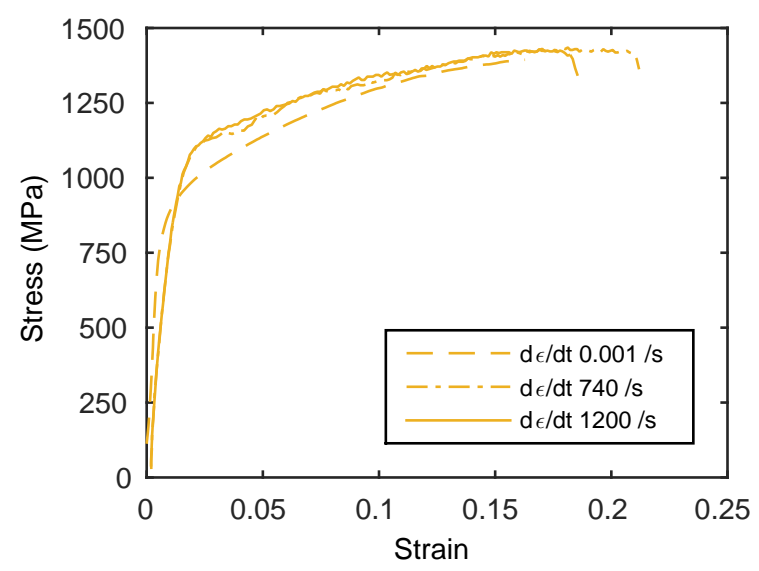

(a)

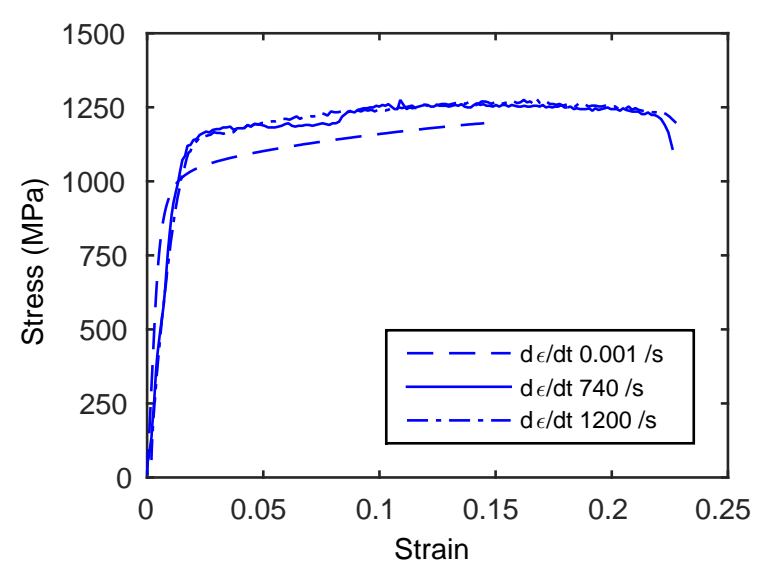

(b)

Fig. 3 Stress-strain curves at different strain rates: (a) $\mathrm{T}=213 \mathrm{~K}$; (b) $\mathrm{T}=298 \mathrm{~K}$

Data variation makes the analysis on the ADI ductility more difficult. However, Fig. 4 indicates that for quasistatic test there is no temperature effect, while the ADI ductility increase with temperature and strain rate under dynamic loading. Same result was reported by Böheme and Reissing [4] also. It is worth noting that, due to the occurrence of necking, reported failure strains in the dynamic regime underestimate the actual values. 


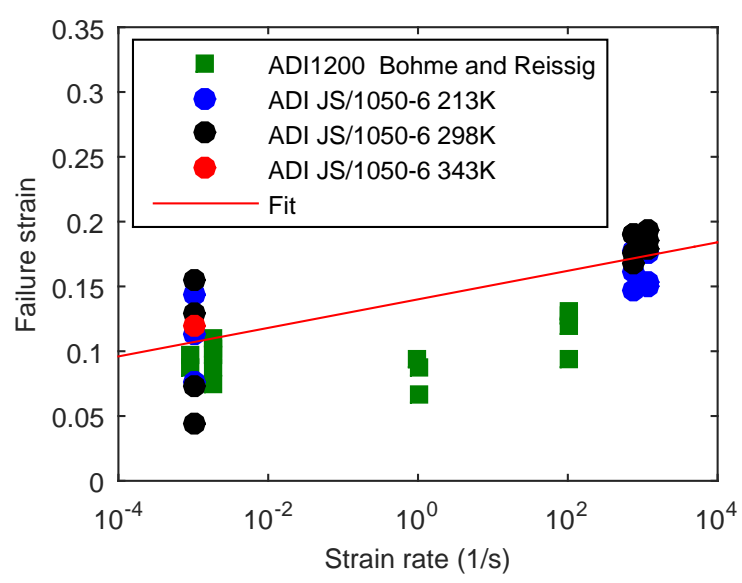

Fig. 4 Failure strain variation with strain rate

\section{Constitutive Modelling Development}

The constitutive model proposed for describing the mechanical response of the ADI JS/1050-6 consists of a yield surface, a hardening relation, and a damage criterion. The first step was the development of a phenomenological strength model able to reproduce the stress-strain curve under different strain rates and temperatures. The following relation was proposed:

$$
\sigma=\left[A(T)\left(1+C \ln \dot{\varepsilon}^{*}\right)+B(T) \varepsilon^{n}\right],
$$

where $\dot{\varepsilon}^{*}=\dot{\varepsilon} / \dot{\varepsilon}_{0}$ is the dimensionless plastic strain rate for $\dot{\varepsilon}_{0}=1.0 \mathrm{~s}^{-1}$. The strain rate term imitates the Johnson and Cook term [5] that well describe the linear variation of the yield stress with the logarithmic of the plastic strain rate. The peculiar, opposite effect of the temperature on yield stress and hardening rate was addressed with the introduction of two temperature dependent functions for the coefficients of the Ludwik's relation (Eq. 1):

$$
\begin{aligned}
& A(T)=A_{S}\left[1-\exp \left(-\frac{T}{m}\right)\right], \\
& B(T)=B_{0} \exp \left(-\frac{T}{m}\right),
\end{aligned}
$$

where $T$ is the absolute temperature in $\mathrm{K}$ and $A_{S}, B_{0}$, and $m$ are material dependent constants.

The second step was the selection of the yield surface function. This need arose from the verification that von Mises criterion does not allow the transferability of the strength model to different states of the stress triaxiality. Numerical simulations of tensile tests using the von Mises criterion overestimate the load-displacement curves, more and more with the triaxiality increase, indicating a pressure dependency of the ADI yield surface. An easy and effective way to account for this behavior is to use the Mohr-Coulomb criterion:

$$
f=\lambda I_{1}+\sqrt{J_{2}}-\frac{\sigma}{\sqrt{3}}=0
$$

Even if the mechanism behind the criterion formulation, of frictional sliding between material particles, is not pertinent to the ADI deformation process, the Mohr-Coulomb criterion has the twofold 
Table 1 Physical properties and coefficients of the strength model and the yield surface function

\begin{tabular}{cccccccc}
\hline$E[\mathrm{MPa}]$ & $\nu$ & $A_{s}[\mathrm{MPa}]$ & $B_{0}[\mathrm{MPa}]$ & $n$ & $m$ & $C$ & $\lambda$ \\
\hline 163000 & 0.25 & 1292 & 9480 & 0.54 & 161 & 0.0148 & 0.058 \\
\hline
\end{tabular}

Table 2 Damage model coefficients for the ADI JS/1050-6

\begin{tabular}{ccccccc}
\hline$\varepsilon_{t h}$ & $\varepsilon_{f}^{0}$ & $d_{1}$ & $d_{2}$ & $T_{m}[\mathrm{~K}]$ & $\alpha$ & $D_{c r}$ \\
\hline 0.04 & 0.145 & 500 & 6.0 & 1500 & 1.0 & 0.1 \\
\hline
\end{tabular}

advantage of being available within finite element (FE) commercial codes and capturing the behavior shown by cast irons.

For nodular cast irons, the pressure dependence is caused by the different morphology of the matrix debonding from the graphite nodule under different stress triaxiality levels [6]. FE-based micromechanical analyses proved to be very effective in giving the resulting macroscopic properties from known characteristics at the microscale [7]. Thus, the unit cell model, developed in [3] for the same ADI, was used to simulate an uniaxial compression test in order to extend data available for the inverse calibration procedure (coefficient $\lambda$ ). The coefficients of the strength model and the yield surface, calibrated on results from tensile tests, are given in Table 1.

Last step was the damage model definition. In DCIs, damage consists of two main mechanisms: the debonding between matrix and spheroids; the nucleation and growth of micro-pores within the matrix. The first leads to the development of a considerable porosity that affects the material strength. This effect has been already taken into account adopting the Morh-Coulomb yield criterion. The second mechanism, due to the limited associated porosity, does not affect the macroscopic strength significantly; however, it does trigger the failure. Damage by nucleation and growth may be effectively addressed with the continuum damage mechanics (CDM) approach [8]. Here, the Bonora's damage model $[9,10]$ was adopted for which damage develops as a function of the total accumulated "active" plastic strain, i.e. the plastic strain accumulated under a positive (tensile) state of stress, according to the following evolution relation:

$$
\dot{D}=\alpha\left[\frac{D_{c r}^{1 / \alpha}}{\ln \left(\varepsilon_{f} / \varepsilon_{t h}\right)}\right] R_{\nu}\left(D_{c r}-D\right)^{(\alpha-1) / \alpha},
$$

where, $\varepsilon_{t h}$ is the threshold strain at which damage processes are activated, $\varepsilon_{f}$ the theoretical failure strain under uniaxial stress at which ductile failure would occur, $D_{c r}$ the critical damage at which failure occurs, and $\alpha$ the damage exponent that controls the shape of damage evolution with plastic strain. The evolution relation account for stress triaxiality level according with following relation:

$$
R_{\nu}=\frac{2}{3}(1+\nu)+3(1-2 \nu)\left(\frac{\sigma_{m}}{\sigma_{e q}}\right)^{2} .
$$

To account for strain rate and temperature effects on the ADI ductility, the following relation for the theoretical uniaxial failure strain was introduced:

$$
\varepsilon_{f}=\left[1+d_{1} \ln \dot{\varepsilon}^{*}\left(\frac{T}{T_{m}}\right)^{d_{2}}\right] \varepsilon_{f}^{0},
$$

where, $T_{m}$ is the melting temperature.

In Fig. 5(a), the failure strains as a function of the stress triaxiality are shown, for two temperatures, together with the failure loci expected for the calibrated model coefficients given in Table 2. Due to the porosity generated by the matrix debonding, the Bridgman's solution to measure the failure strain from post mortem fracture surface diameter is no longer accurate. Indeed, failure strain for each test 
was determined as the value calculated by FEM for the displacement at which fracture is observed in the experiments. The associated stress triaxiality was calculated as the mean value over the strain path, between $\varepsilon_{t h}$ and $\varepsilon_{f}$, for the point where failure is expected to occur first. In Fig. 5 (b), the variation of $\varepsilon_{f}$ with strain rate as expected by the proposed model (Eq. 7) is compared with experimental results. In Fig. 6 the comparisons between experimental and calculated stress-strain curves are given for both quasistatic and dynamic conditions. These demonstrate the capability of the proposed constitutive model in reproducing the ADI behavior under different conditions of strain rate and temperature in terms of both flow stress response and failure strain.

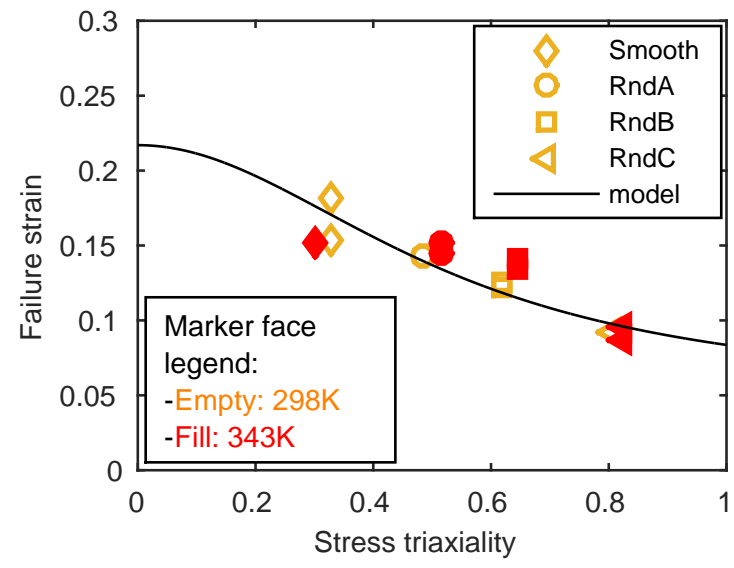

(a)

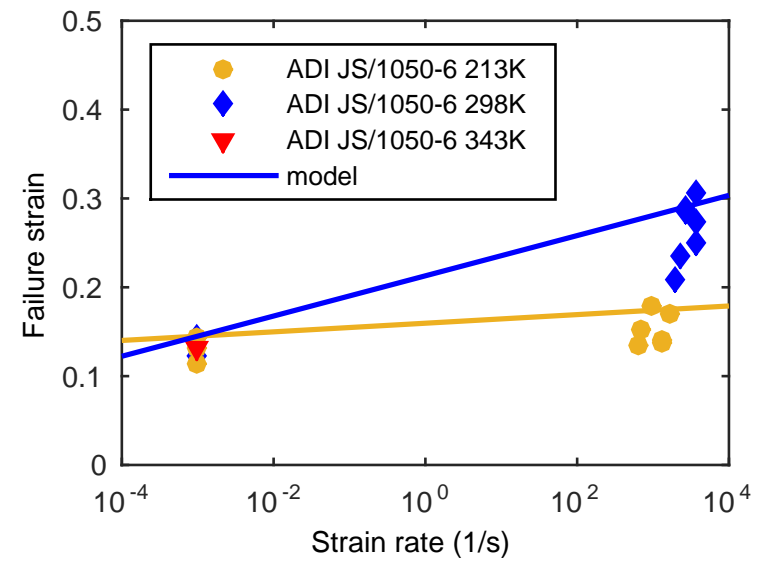

(b)

Fig. 5 Failure strain variation with: (a) stress triaxiality level; (b) strain rate

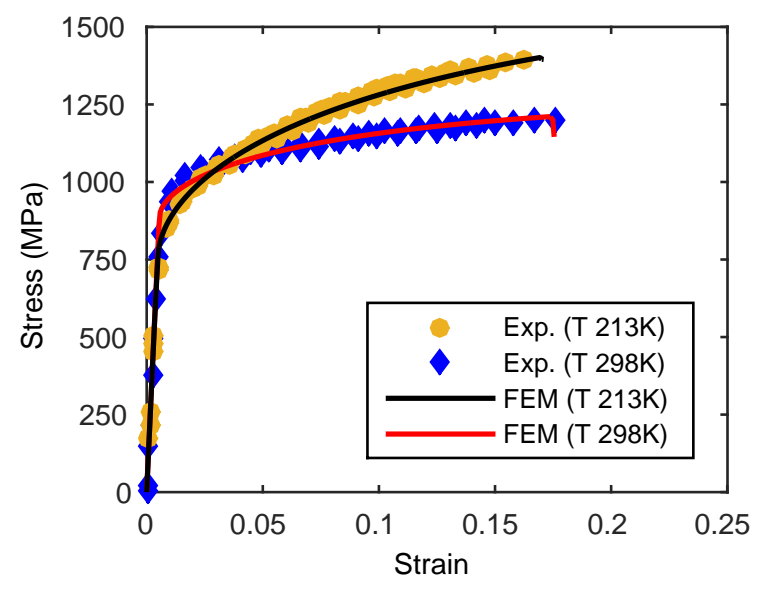

(a)

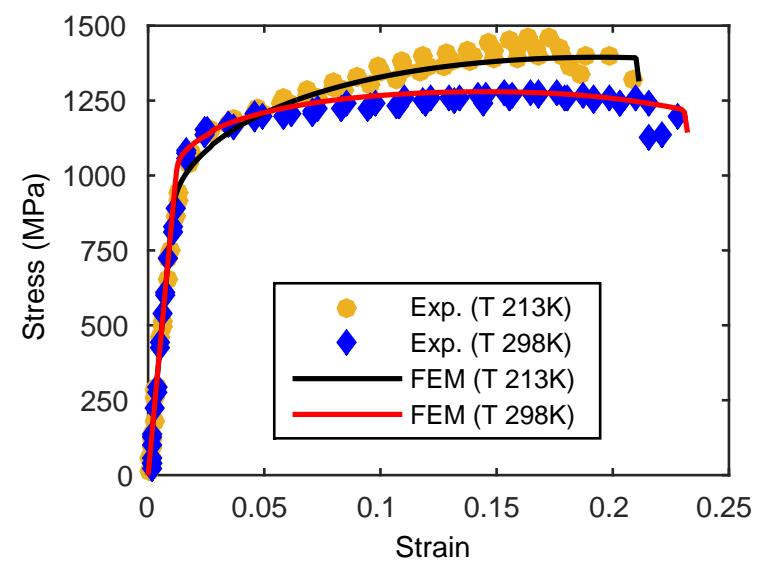

(b)

Fig. 6 Comparison between experimental and calculated stress-strain curves: (a) quasistatic conditions; (b) high strain rate $(1200 / s)$

\section{Concluding Remarks}

The results of the experimental tests performed on the ADI ISO 17804/JS/1050-6/S can be summarized, from a macroscopic point of view, as follows:

- an increase of the temperature entails a decrease of the hardening rate and a slightly increase of the yield stress; 
- at high strain rate, temperature increases ductility while no effect is observed under quasistatic conditions;

- an increase of the strain rate entails an increase of both the yield strength and the failure strain;

- the pressure affects the yield surface.

The observed features were taken into account in the formulation of a constitutive model aimed to be a simple and functional design tool. The pressure effect on the yield surface was accounted for by adopting the Mohr-Coulomb yield function. Strain rate and temperature effects on the material strength required the formulation of a new hardening relation. Finally, the Bonora's damage model was selected to predict failure under different stress triaxiality levels. A strain rate and temperature dependence of one of the damage model coefficients $\left(\varepsilon_{f}\right)$ was introduced to account for the observed increase of ductility.

Simulations of the tensile tests demonstrated the capability of the model in reproducing the experimental results with good accuracy.

\section{References}

[1] F. Zanardi, F. Bonollo, G. Angella, N. Bonora, G. Iannitti, A. Ruggiero, A contribution to new material standards for ductile irons and austempered ductile irons, Int. J. Metalcast. 11 (2017) 136-147.

[2] F.J. Zerilli, R.W. Armstrong, Dislocation-mechanics-based constitutive relations for material dynamics calculations, J. Appl. Phys. 61 (1987) 1816-1825.

[3] G. Iannitti, A. Ruggiero, N. Bonora, S. Masaggia, F. Veneri, Micromechanical modelling of constitutive behavior of austempered ductile iron (ADI) at high strain rate, Theor. Appl. Fract. Mec. 92 (2017) 351-359.

[4] W. Böhme, L. Reissig, Capability of new high strength ADI-Materials for automotive components under crash loading, Adv. Eng. Mater. 17 (2015) 1189-1196.

[5] G.R. Johnson, W.H. Cook, A constitutive model and data for metals subjected to large strains, high strain rates and high temperatures, Proc. of the Seventh Int. Symp. on Ballistics 21 (1983) 541-547.

[6] A. Ruggiero, N. Bonora, D. Gentile, G. Iannitti, G. Testa, M. Hörnqvist Colliander, S. Masaggia, F. Vettore, Strain rate effects on fracture behavior of austempered ductile irons, submitted to AIP Conf. Proc. (2017).

[7] N. Bonora, A. Ruggiero, Micromechanical modeling of ductile cast iron incorporating damage. part i: Ferritic ductile cast iron, Int. J. Solids. Struct. 42 (2005) 1401-1424.

[8] J. Lemaitre and J.L. Chaboche, Mechanics of solid materials, Cambridge university press, 1994.

[9] N. Bonora, A nonlinear cdm model for ductile failure, Eng. Fract. Mech. 58 (1997) 11-28.

[10] A. Pirondi, N. Bonora, Modeling ductile damage under fully reversed cycling, Comp. Mater. Sci. 26 (2003) 129-141. 